\title{
Mapeo de la economía social y solidaria en el municipio de Santa Fe (Argentina): creación de una base de datos actualizada de las diferentes experiencias
}

\author{
Lucas Gabriel Cardozo*, Eugenio Serafino ${ }^{\star *}$, Orlando Sotto ${ }^{\star * *}$, Julio Claudio Tealdo****
}

* Maestrando en Economía Social, Universidad Nacional General Sarmiento. Miembro del Programa de Extensión de Economía Social y Solidaria, Universidad Nacional del Litoral, Santa Fe, Argentina. Correo electrónico: 1cardozo@fcjs.unl.edu.ar

** Licenciado en Administración de Empresas. Miembro del Programa de Extensión de Economía Social y Solidaria y docente, Universidad Nacional del Litoral, Santa Fe, Argentina. Correo electrónico: eserafino@unl.edu.ar

*** Licenciado en Administración de Empresas. Diplomado en Desarrollo Local y Economía Social. Miembro del Programa de Extensión de Economía Socia y Solidaria y docente, Universidad Nacional del Litoral, Santa Fe, Argentina. Correo electrónico: sottoorlando@gmail.com

**** Licenciado en Administración. Diplomado en Desarrollo Local y Economía Social. Director del Programa de Extensión de Economía Social y Solidaria, y docente titular, Universidad Nacional del Litoral, Santa Fe, Argentina. Correo electrónico: jctealdo@fcjs.unl.edu.ar

Recibido: 17 de julio del 2016

Aprobado: 7 de noviembre del 2016

Cómo citar este artículo: Cardozo, L. G., Serafino, E., Sotto, 0. y Tealdo, J. C. (2017). Mapeo de la economía social y solidaria en el municipio de Santa Fe (Argentina): creación de una base de datos actualizada de las diferentes experiencias. Cooperativismo \& Desarrollo, 110(25), 05-15. doi: http://dx.doi. org/10.16925/co.v25i110.1760

\section{Resumen}

Propósito: presentar un mapeo socioterritorial de las expresiones de la economía social y solidaria localizadas en Santa Fe. Temas: para la realización del artículo, se consultó bibliografía específica en la temática de la economía social y solidaria, y las distintas fuentes en las que se destaca la importancia y los relevamientos llevados a cabo en países de América Latina. Desarrollo: para elaborar los mapas se recurrió a la consulta de fuentes secundarias que proporcionaron los datos correspondientes para luego procesarlos y representarlos a través de un sistema de información geográfica. Conclusiones: los resultados que arroja el artículo se constituyen en la primera fuente de información, teniendo en cuenta que no existe un trabajo de esta característica para el territorio en cuestión. Este artículo es una base importante para dilucidar la situación de las viejas y de las nuevas tradiciones de la economía social y solidaria con los referentes clave de Santa Fe. Se espera que los resultados obtenidos se utilicen como plataforma para diseñar e implementar diversas propuestas de trabajo a fin de resolver problemáticas de las experiencias involucradas en el campo de las políticas públicas.

Palabras clave: Argentina, cartografía, economía social y solidaria, municipio de Santa Fe. 


\title{
Mapping the Social and Solidarity Economy in the Municipality of Santa Fe (Argentina): Creation of an updated Database of the Different Experiences
}

\begin{abstract}
Purpose: The paper aims to present a socio-territorial mapping of the expressions of social and solidarity economy in Santa Fe. Topics: Specific bibliography about social and solidarity economy was consulted, as well as different sources that emphasize on the importance and on studies and surveys carried out in Latin American countries. Development: To draw up the maps secondary sources that provided the corresponding data were consulted. The data was then processed and represented with a geographic information system. Conclusions: The results obtained constitute a first and primary source of information, taking into account that there is no previous work with these characteristics for the territory in question. This article is an important basis for elucidating the situation of old and new traditions of the Social and Solidarity Economy with the key referents of Santa Fe. It is expected that the obtained results will be used as a platform to design and implement various work proposals to solve problems of the experiences given in the field of public policies.
\end{abstract}

Keywords: Argentina, cartography, social and solidarity economy, municipality of Santa Fe.

\section{Mapeamento da economia social e solidária no município de Santa Fe (Argentina): criação de uma base de dados atualizada das diferentes experiências}

\section{Resumo}

Propósito: apresentar um mapeamento socioterritorial das expressões da economia social e solidária em Santa Fe. Temas: para a realização do artigo, consultou-se a bibliografia específica na temática da economia social e solidária, e as diferentes fontes em que se destaca a importância e os levantamentos realizados em países da América Latina. Desenvolvimento: para elaborar os mapas, recorreu-se à consulta de fontes secundárias que proporcionaram os dados correspondentes para, em seguida, processá-los e representá-los por meio de um sistema de informação geográfica. Conclusões: os resultados apresentados no artigo constituem a primeira fonte de informação considerando que não existe um trabalho dessa característica para o território em questão. Este artigo é uma base importante para esclarecer a situação das velhas e das novas tradições da economia social e solidária com os referentes-chave de Santa Fe. Espera-se que os resultados obtidos sejam utilizados como plataforma para desenhar e implantar diversas propostas de trabalho para resolver problemáticas das experiências envolvidas no campo das políticas públicas.

Palavras-chave: Argentina, cartografia, economia social e solidária, município de Santa Fe. 


\section{Introducción ${ }^{1}$}

Desde hace más de una década, asistimos a una explosión de la economía social y solidaria (Esys) en Argentina, en la cual conviven las "viejas" tradiciones y las "nuevas" tradiciones. Comprendiendo en las primeras a las experiencias históricas del cooperativismo y el mutualismo, y en las segundas, los emprendimientos socioproductivos, las iniciativas económicas de los movimientos de desocupados, las diferentes experiencias de finanzas solidarias, las empresas "recuperadas" por sus trabajadores, las formas de intercambio equitativo y de monedas sociales, entre otras (Pastore, 2010).

En la actualidad, la Esys ha cobrado importancia en el plano de las prácticas con la generación de otra forma de entendimiento de lo económico, no solo en el plano material, sino en el simbólico. Este resurgimiento se instauró como un campo de lucha para los sectores populares, que a partir de cambios culturales, económicos y políticos se organizaron para generar nuevos espacios asociativos, posteriores a la crisis del 2001, para el caso argentino (para una lista de las nuevas formas de emergencia de emprendimientos véase Abramovich y Vázquez, 2007). De esta manera, se generó una clara diferenciación de las cooperativas y mutuales de larga data que han sido resignificadas, no solo por el poder de la autogestión de los trabajadores, sino también por el accionar del Estado a través de la formulación de políticas sociales y de empleo que fomentan la conformación de cooperativas de trabajo (Vuotto, 2007).

Siguiendo esta línea, Susana Hintze (2007 y 2013) asegura que la construcción de políticas públicas para este sector permite la intersectorialidad, la transversalidad y la territorialización entre los diferentes organismos del Estado, en sus diferentes escalas de actuación y en conjunto con la sociedad. Por lo tanto, el conocimiento de las diferentes expresiones de Esys en el ámbito socioterritorial se torna de gran importancia: en primer lugar, porque es necesario cuantificar las experiencias y conocer su

Resultados parciales del presente trabajo fueron presentados en dos congresos. El primero bajo la misma autoría: Cardozo, Serafino, Sotto y Tealdo (2015), y el segundo por Cardozo y Serafino (2015). El artículo forma parte del proyecto de investigación "Curso de Acción para la Investigación y Desarrollo 'Mapeo, caracterización e interacción de los actores de la Economía Social y Solidaria en los municipios de Santa Fe, Rosario y Reconquista", dirigido por Julio Tealdo, aprobado por Resolución del Consejo Superior de la Universidad Nacional del Litoral N. ${ }^{\circ} 223 / 15$. Por último, los autores agradecen a Eduardo Rabellino por la elaboración de los mapas. conformación; en segundo lugar, para saber sobre su producción y, en tercer lugar, localizarlas para determinar su ubicación y de esta manera contextualizar a partir de la información territorial.

En Santa Fe, Argentina, en los últimos años, asistimos a una multiplicidad de experiencias que han surgido y que hoy son relevadas y potenciales beneficiarios de políticas de Esys. Esta situación nos invita a preguntarnos: ¿¿cuántas experiencias de este tipo existen fehacientemente en el municipio?, ¿qué producen?, ¿en qué lugar se encuentran? Por último, ¿para qué sirve dilucidar estos interrogantes?

Para responder a los interrogantes, el presente artículo se encuentra estructurado de la siguiente manera: en primer lugar, presentamos una breve mención de los estudios de mapeos que se llevaron a cabo en Latinoamérica que consideramos importantes para analizar y referenciar a la hora de profundizar sobre la temática. Seguidamente, comentaremos la metodología utilizada y el objetivo propuesto. Continuando con el trabajo, elaboramos una pequeña contextualización de la situación actual de las experiencias de la Esys en Santa Fe, complementariamente abordaremos un análisis desagregado de la información y la producción cartográfica. Por último, vienen las consideraciones finales en términos propositivos para seguir profundizando los resultados que se difunden en el artículo.

\section{Mapear la Esys: antecedentes en la temática}

En un reciente artículo, Luiz Gaiger (2014) nos invita a conocer globalmente los estudios de la Esys. Este llamado resulta interesante y desafiante, especialmente porque el autor señala la importancia que asume el mapeo de las diferentes experiencias que engloba a la Esys por parte de los diversos grupos de investigación de las universidades y del Estado en sus distintas escalas de actuación. La medición se vuelve una herramienta estratégica para América Latina, porque ayuda a tomar conocimiento de las experiencias y a fortalecer dichos espacios identificando los actores que participan de las formas que adopta la Esys. Además, el mapeo de la esys nos permite asumir desafíos metodológicos en la tarea de relevar aspectos relacionados con la información cuantitativa y también con la cualitativa para la construcción de una base de datos que permita analizar a la Esys en los diferentes territorios. En este sentido, la cartografía nos ayuda a tener una idea sobre la situación locacional y el reconocimiento para establecer futuras intervenciones. 
Como adelantamos, el tema del mapeo no es algo nuevo en la Esys. Como antecedentes, se puede destacar los trabajos que se formularon para recabar información en Latinoamérica, el Caribe y en Canad ${ }^{2}$. A continuación, listaremos las principales contribuciones que se realizaron en Latinoamérica, destacando el grupo de trabajo, así como las instituciones que contribuyeron para la recopilación de la información en los diferentes territorios.

La guía realizada por Coraggio, Arancibia y Deux (2010) nos ofrece un marco teórico, así como las experiencias llevadas a cabo en Argentina y Brasil. Para el caso argentino, los autores comentan las dos experiencias desarrolladas por el Instituto Nacional de Asociativismo de Economía Social (Inaes) y la Universidad Nacional Tres de Febrero (UNTREF) en el 2007. La muestra fue el sector "tradicional" de la economía social, es decir, las cooperativas y mutuales. El segundo trabajo fue el que estuvo organizado por el Instituto del Conurbano de la Universidad Nacional General Sarmiento (ICO-UNGS) en colaboración con la Secretaría de Políticas Sociales y Desarrollo Humano del Ministerio de Desarrollo Social. El objeto de estudio estuvo focalizado en los emprendimientos socioeconómicos asociativos, ampliando la propuesta de trabajo a diferentes universidades argentinas.

En el caso brasilero, entre el 2005 y el 2006, se realiza el Mapeamento da Economia Solidária, tarea llevada a cabo por el Ministerio do Trabalho e Emprego de la Nación, Secretaria Nacional de Economia Solidária, Observatório di Mercado do Trabalho, Forum da Economia Solidária, representantes de emprendimientos económicos y entidades de apoyo al sector. El mapeo tuvo mostró la existencia de 21578 emprendimientos solidarios (Coraggio, Arancibia y Deux, 2010). La segunda mensura se puede consultar en el Atlas Digital de Economía Solidária, información construida con los datos del Segundo Mapeamento Nacional de Emprendimentos Econômicos Solidários (EES), realizado por la Secretaria Nacional de Economia Solidária, finalizado en el $2013^{[3]}$. Con

\footnotetext{
2 En Quebec (Canadá), Vaillancourt (2010) analiza las experiencias que se desarrollaron en el periodo de más de una década. El autor divide las tareas desarrolladas en dos cohortes temporales. La primera del 2000-2005, cuando intervinieron varios actores: instituciones universitarias, gobierno y asociaciones de la sociedad civil. La segunda, corresponde a los años 2006-2011; el trabajo realizado se puede encontrar en el Canadian Social Economy HuB y el mapeo es en el ámbito nacional: allí se puede observar la heterogeneidad de la Esys canadiense.

3 Para mayor información se puede consultar: http://sies.ecosol.org.br/atlas
}

la participación del grupo de Pesquisa em Economia Solidária e Cooperativa da Unisinos, Rilles y el CNPq, entre otros. Como resultado de este trabajo se presentó el libro As faces da Economia Solidária no Brasil.

Otra de las experiencias de mapeo es la propuesta de Uruguay. Como lo indica la web:

El mapeo tiene por objetivo identificar, estudiar y ubicar en el territorio las experiencias económicas de Economía Solidaria (EcSol) que se dan en Uruguay, tanto a nivel productivo (emprendimientos), como en la distribución (tiendas, redes de comercialización), el consumo (colectivos de consumidores), las finanzas (finanzas solidarias). También visualizar las entidades de apoyo o fomento de estas experiencias y estudiar el vínculo con ella ${ }^{4}$.

La propuesta de la medición está a cargo de la Universidad de la República a través de la Unidad de Estudios Cooperativos (UEC) desde el 2014.

Para finalizar el recorrido de los mapeos, vamos a hacer referencia al caso argentino. Señalamos dos obras, uno es el libro publicado por Coraggio y Sabaté (2010) con base en una encuesta realizada en diferentes puntos de nuestro país. Las hipótesis del relevamiento se basan en que la lógica de la economía de los emprendimientos está estrechamente vinculada a la reproducción de las unidades domésticas y a las comunidades; estas no existen aisladas de redes y articulaciones más amplias que superan la relación exterior de intercambio en un mercado. Se relevaron organizaciones y emprendimientos que sean potenciales componentes de redes consolidadas de economía social y solidaria, siendo estas la plataforma de acción compleja para transitar hacia estructuras de organización de producción y reproducción centradas en el trabajo y orientadas a la reproducción ampliada de la vida de todos.

Recientemente, Colombari y Molina (2014) presentan el mapeo del Observatorio Social sobre Empresas Recuperadas y Autogestionada (Osera), el cual se realizó con los datos del Ministerio de Trabajo, Empleo y Seguridad Social. La escala de cobertura del mapa es nacional y cuenta con información actualizada por el equipo interdisciplinario de trabajo del Osera ${ }^{5}$.

\footnotetext{
4 Para mayor información se puede consultar: http://mapeoecsol.blogspot.com.ar/

5 Para ampliar información visitar: http://empresasrecuperadas.org/mapa.php
} 
Estos trabajos disponen de gran información y pueden ser utilizados como referencia para ampliar el presente estudio que, como hemos mencionado, se trata de acercarnos a una temática novedosa y de tipo exploratorio a través de fuentes secundarias. En el apartado siguiente mencionaremos las consideraciones correspondientes sobre la metodología utilizada.

\section{Breves consideraciones metodológicas}

Para el presente trabajo hemos utilizado fuentes de información secundarias obtenidas del Inaes del Ministerio de Desarrollo Social de la Nación y de la Dirección de Economía Social de la Municipalidad de Santa Fe. Una vez reveladas, se procedió a la tabulación, el análisis y la categorización de las diferentes experiencias de la esys. Seguidamente, obtenida la información necesaria, la procesamos en un Sistema de Información Geográfica (SIG) en el cual las localizamos georreferenciadamente y obteniendo como resultado mapas temáticos.

En términos de referencias temporo-espaciales, se obtuvo el relevamiento de las cooperativas, mutuales y emprendimientos registradas hasta el 2014 inclusive, localizadas en Santa Fe, provincia de Santa Fe, Argentina.

El objetivo principal es crear y presentar un mapeo socioterritorial que comprenda las expresiones de la Esys localizadas en Santa Fe como primera aproximación a un diagnóstico situacional. Cabe mencionar que el presente estudio es preliminar ${ }^{6} ; \sin$ embargo, permite sentar las bases para posteriores trabajos de profundización y relevamiento vinculados a la temática teniendo en cuenta que a escala local no se han procurado trabajos con los resultados que se presentan en el artículo.

\section{Mapas de la Esys a escala local: resultados obtenidos en el municipio de Santa Fe}

El distrito de Santa Fe cuenta con 525093 habitantes (Indec, 2010), conformándose como uno de los distritos más poblados del país. En dicho municipio aún no existe un registro actualizado y unificado de Esys. Si bien se llevaron a cabo diferentes relevamientos, la actualización de este que contemple las diferentes

\footnotetext{
6 Como hemos mencionado, se inscribe en un proyecto de investigación que se encuentra en plena ejecución y plantea la comparación con otros dos municipios del territorio provincial: Rosario y Reconquista.
}

tradiciones de esys todavía no se encuentra disponible, lo cual es de suma importancia para el diseño e implementación de políticas públicas.

Si tenemos en cuenta las "viejas" tradiciones, la provincia de Santa Fe históricamente ha sido una de las provincias argentinas en las que más se ha propiciado la constitución y el desarrollo de mutuales y cooperativas. El origen de estas se visualiza, fundamentalmente, en la gran influencia de inmigrantes europeos que han poblado la zona agrícola-ganadera, donde los servicios y prestaciones que proveían este tipo de organizaciones reemplazaban, en muchas ocasiones, al Estado (Begeneta, 2015).

En cuanto a las "nuevas" tradiciones en las grandes aglomeraciones urbanas como Santa Fe, con sus particularidades, no han quedado ajenas a estos procesos asociativos: sobresale en los últimos años la proliferación de cooperativas de trabajo como respuesta a la problemática de la desocupación. Una posible solución a esta situación en los diferentes niveles del Estado han sido las "nuevas experiencias" de la Esys que se multiplicaron como una alternativa, fundamentalmente para mujeres y jóvenes excluidos del mercado laboral "tradicional" (Tealdo, Sotto y Serafino, 2015).

Santa Fe, como desarrollaremos en el presente trabajo, no ha sido la excepción en cuanto al surgimiento y constitución de dichas experiencias. En este sentido, las cartografías de estas, su identificación y su localización se tornan indispensables para la construcción de un sistema de información a nivel socioterritorial y para la definición de las políticas públicas vinculadas a la Esys.

A continuación, presentaremos el análisis de los datos recopilados y procesados de las cooperativas, mutuales y emprendimientos productivos y su cartografía correspondiente en Santa Fe.

\section{Composición de las "viejas" y "nuevas" tradiciones de la Esys en el municipio}

Partiendo del análisis en conjunto, el 77\% de las experiencias de Esys en Santa Fe están constituidas por emprendimientos productivos ${ }^{7}$, las cooperativas

Cabe destacar que el crecimiento de los emprendimientos productivos ha sido muy importante en los últimos años, llegando a duplicarse en poco menos de dos años (2012-2014). Si bien el relevamiento fue realizado con fuentes secundarias, los responsables de la Dirección de Economía Social de la Municipalidad de Santa Fe compartieron con el equipo de trabajo información según la cual, en el 2011 había inscriptos en el Registro de Emprendedores Productivos Locales aproximadamente 350 emprendimientos productivos, siendo hasta principios del 2015, 859 iniciativas productivas. 
se ubican en un segundo lugar con un $14 \%$ y, por último, las mutuales con un 9\%. En la tabla 1, se puede observar los guarismos en términos absolutos y relativos de las experiencias enunciadas.

Tabla 1

Composición de experiencias de la Esys en Santa Fe

\begin{tabular}{|l|c|c|}
\hline \multicolumn{1}{|c|}{ Experiencias de la Esys } & Cantidad & $\%$ \\
\hline Cooperativas & 154 & $14 \%$ \\
\hline Mutuales & 106 & $9 \%$ \\
\hline Emprendimientos productivos & 859 & $77 \%$ \\
\hline Total & 1119 & $100 \%$ \\
\hline
\end{tabular}

Nota. Elaboración propia con base en datos del Registro de Emprendedores de la Municipalidad de Santa Fe y el registro del Inaes del 2014.

\section{Las Cooperativas en el municipio}

Como hemos analizado en la tabla 1, la actividad cooperativa en el municipio ocupa el $14 \%$ en el total de las experiencias solidarias siendo 154 cooperativas en números absolutos. A su vez, establecimos las diferentes categorías que conforman el núcleo cooperativo a partir de las actividades que estas realizaron. De acuerdo con su objeto social, se clasifican en: agropecuarias; construcción de viviendas; trabajo y provisión de servicios (tabla 2).

Tabla 2

Porcentajes de cooperativas por actividad en Santa Fe, 2014

\begin{tabular}{|l|c|c|}
\hline \multicolumn{1}{|c|}{ ACTIVIDAD COOPERATIVA } & CANTIDAD & $\%$ \\
\hline Agropecuaria & 1 & 0,64 \\
\hline Vivienda & 4 & 2,6 \\
\hline Trabajo & 135 & 88 \\
\hline Provisión-Servicios Públicos & 14 & 8,76 \\
\hline Total & 154 & 100 \\
\hline
\end{tabular}

Nota. Elaboración propia con base en INAES, 2014.

Si desagregamos el universo de cooperativas del municipio, se puede observar, en la figura 1, que el porcentaje mayor de las actividades que realizan las iniciativas asociativas se encuentra representado por las cooperativas de trabajo, que representan un $88 \%$; seguidamente, y con amplia diferencia se encuentran las cooperativas de provisión de servicios públicos, con un $9 \%$; existe un $2 \%$ de las de viviendas, y un $1 \%$ de agropecuaria.

Un dato para destacar es que el total de las cooperativas de trabajo registradas es de 135 , con la particularidad de que el $97 \%$ se constituyeron en el presente siglo, luego de la crisis económica-política del 2001; hasta dicho año solo existían en Santa Fe cuatro cooperativas de trabajo.

En la figura 2, se puede apreciar que la distribución del total de las cooperativas es dispersa, es decir, en todo el territorio y, en términos generales, existen dos polos de concentración, en la zona norte y sur del municipio.

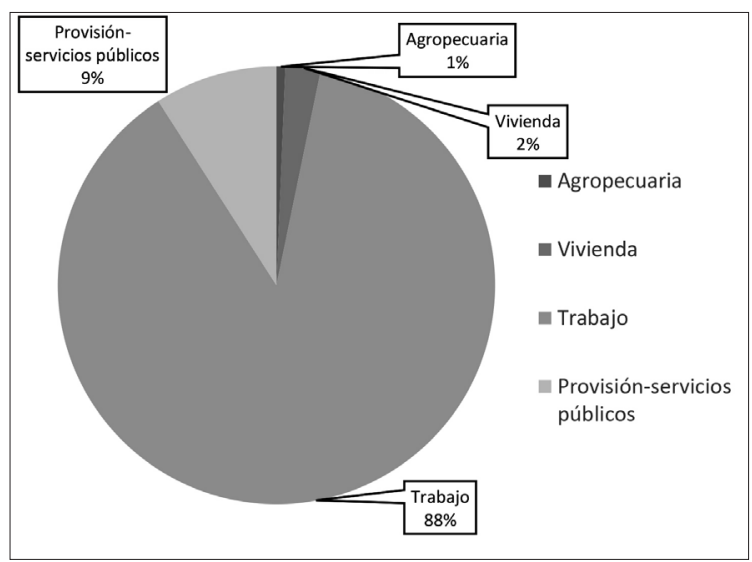

Figura 1. Porcentaje de cooperativas del municipio de Santa Fe por actividad, 2014. Elaboración propia con base en datos del INAES.

En la figura 2 se puede visualizar la distribución de estas en el ámbito territorial del municipio.

\section{Las mutuales en el municipio}

Siguiendo con el análisis, en la tabla 3 se ve que aproximadamente un $54 \%$ de las mutuales registradas en el territorio santafesino pertenecen al rubro de "otras-sin denominación" (otras-s/D), seguidas por las de "salud, asistencia y servicios sociales" y, en menor medida, por las de "ayuda económica, ahorro, crédito y subsidios". Las mutuales dedicadas al fomento de la cultura y a provisión, consumo y vivienda representan porcentajes insignificantes.

Con respecto a la primera categoría enunciada (otras-s/D), nos interpela a identificar en un futuro 


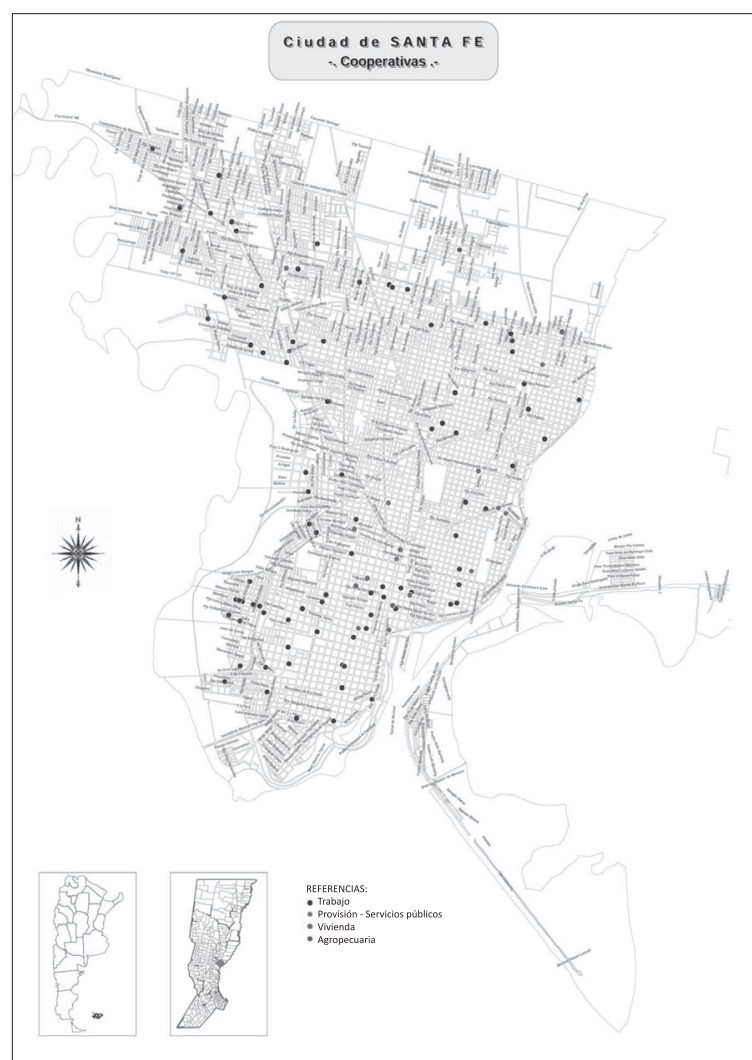

Figura 2. Distribución territorial de cooperativas en Santa Fe. Elaboración propia con base en datos del INAES.

trabajo de campo su objeto social, y de esta manera, poder diversificar el padrón de registro de acuerdo a la actividad que realizan. En la figura 4 , se puede observar que la mayoría de las mutuales se encuentran concentradas en un área, la misma corresponde al área comercial del microcentro del municipio.

Tabla 3

Porcentaje de Mutuales por actividad en Santa Fe en el 2014

\begin{tabular}{|l|c|c|}
\hline \multicolumn{1}{|c|}{ Actividad Mutual } & Cantidad & $\%$ \\
\hline $\begin{array}{l}\text { Ayuda económica, ahorro, crédito y } \\
\text { subsidios }\end{array}$ & 16 & 15,09 \\
\hline Salud, asistencia y servicios sociales & 24 & 22,64 \\
\hline Provisión, consumo y vivienda & 6 & 5,66 \\
\hline Cultura & 3 & 2,83 \\
\hline Otras-S/D & 57 & 53,78 \\
\hline Total & 106 & 100 \\
\hline
\end{tabular}

Nota. Elaboración propia con base en datos del INAES.

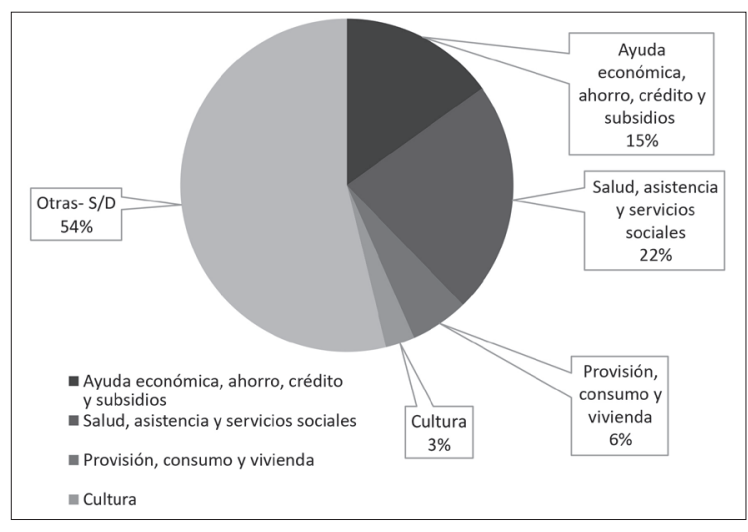

Figura 3. Mutuales de Santa Fe por actividad, 2014. Elaboración propia con base en datos del INAES.

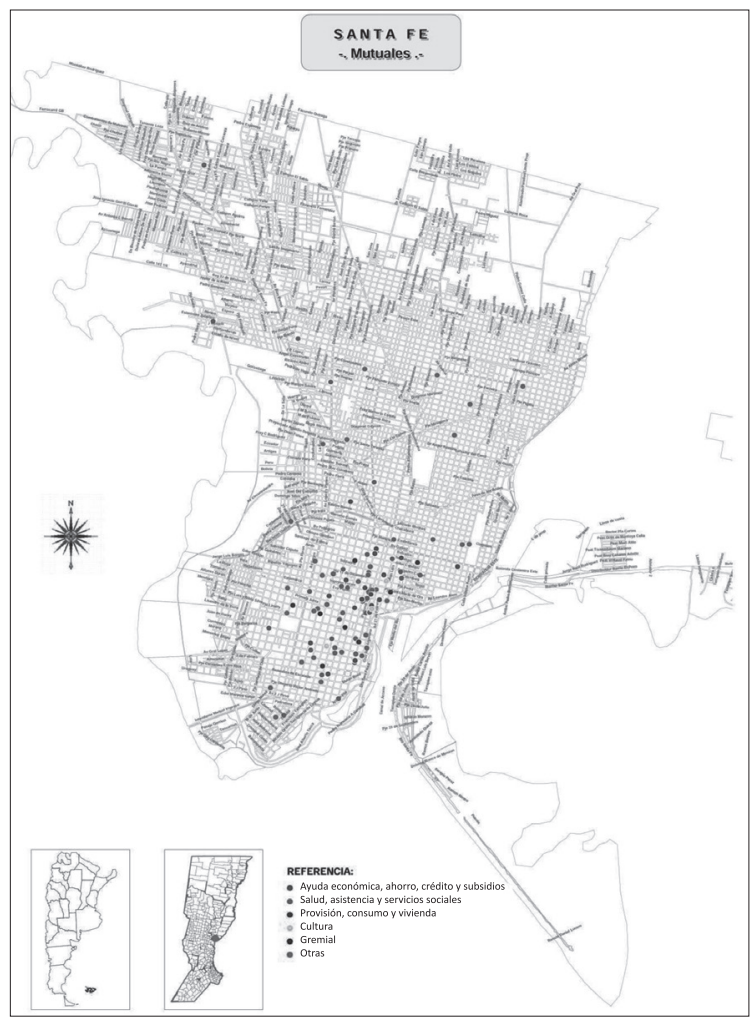

Figura 4. Distribución territorial de mutuales en Santa Fe. Elaboración propia con base en datos del INAES.

\section{Los emprendimientos productivos del municipio}

Estas experiencias representan el 77\% del total de prácticas de Esys del territorio santafesino. Como se puede apreciar en la tabla 4, estos poseen mayores rubros con relación a los dos casos que fueron desarrollados con anterioridad. Dichos rubros se dividen en siete categorías: 
"alimenticio", “artesanías", “carpintería”, "herrería”, “textil”, "plantas", "servicios” y "otros".

Tabla 4

Porcentaje de emprendimientos productivos por rubro en Santa Fe, 2014

\begin{tabular}{|l|c|c|}
\hline \multicolumn{1}{|c|}{ Rubro } & Cantidad & \% s/el Total \\
\hline Alimenticio & 80 & 9,31 \\
\hline Artesanías & 259 & 30,15 \\
\hline Carpintería & 10 & 1,16 \\
\hline Herrería & 21 & 2,44 \\
\hline Textil & 180 & 20,95 \\
\hline Plantas & 3 & 0,3 \\
\hline Servicios & 108 & 12,57 \\
\hline Otros & 198 & 23,12 \\
\hline Total & 859 & 100 \\
\hline
\end{tabular}

Nota. Elaboración propia con base en datos del Registro de Emprendedores de la Municipalidad de Santa Fe.

El total de los emprendimientos en números absolutos es de 859 y, como se muestra en la figura 5, el rubro de "artesanías" es el que ocupa el mayor porcentaje con un $30 \%$. En el rubro "otros", cuyo porcentaje es el segundo mayor con un $23 \%$, se agrupan una multiplicidad de emprendimientos destinados a diferentes actividades económicas (peluquería, viveros, diarios y revistas y mercerías, por mencionar algunos). La actividad textil se encuentra en tercer lugar con un $21 \%$; un 13\% representa las actividades de servicios, y, por último, con un $9 \%$ está la categoría de alimentos. Estos datos pueden visualizarse en la figura 5 .

En cuanto a la distribución territorial podemos observar que los emprendimientos productivos se encuentran distribuidos por todo el territorio municipal, con cual se podría realizar un trabajo de formación de redes de emprendimientos de acuerdo con las categorías y también generar entramados productivos solidarios que permitan consolidar las diferentes etapas de los diversos procesos: producción, distribución, circulación, financiamiento y consumo. En este sentido, creemos necesario destacar que se está trabajando con dichas experiencias en el Centro de Emprendedores de Economía Social de Santa Fe.

Lozeco (2015) describe y desarrolla el Centro de Emprendedores de Economía Social, espacio de articulación de políticas públicas vinculadas a la economía social. Esta es una iniciativa que conecta tres escalas de gobierno: el municipio de Santa Fe, el Gobierno provincial y la Universidad Nacional del Litoral. A través

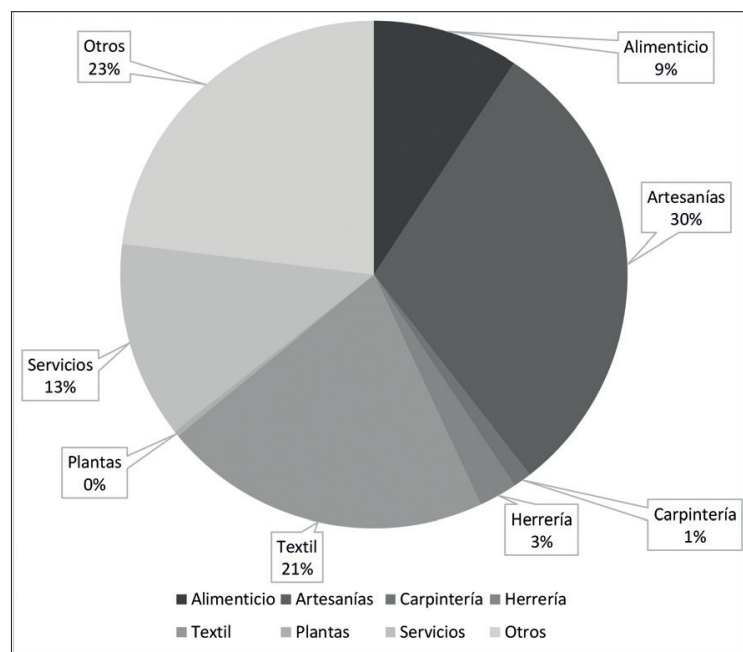

Figura 5. Emprendedores productivos de Santa Fe por Rubro, 2014. Elaboración propia con base en datos del Registro de Emprendedores de la Municipalidad de Santa Fe.

de la concertación de trabajo de estos tres actores se desarrollan actividades con el fin de que los emprendimientos productivos puedan recibir formación, logren dejar la individualidad y comiencen a asociarse con otros emprendimientos del mismo rubro.

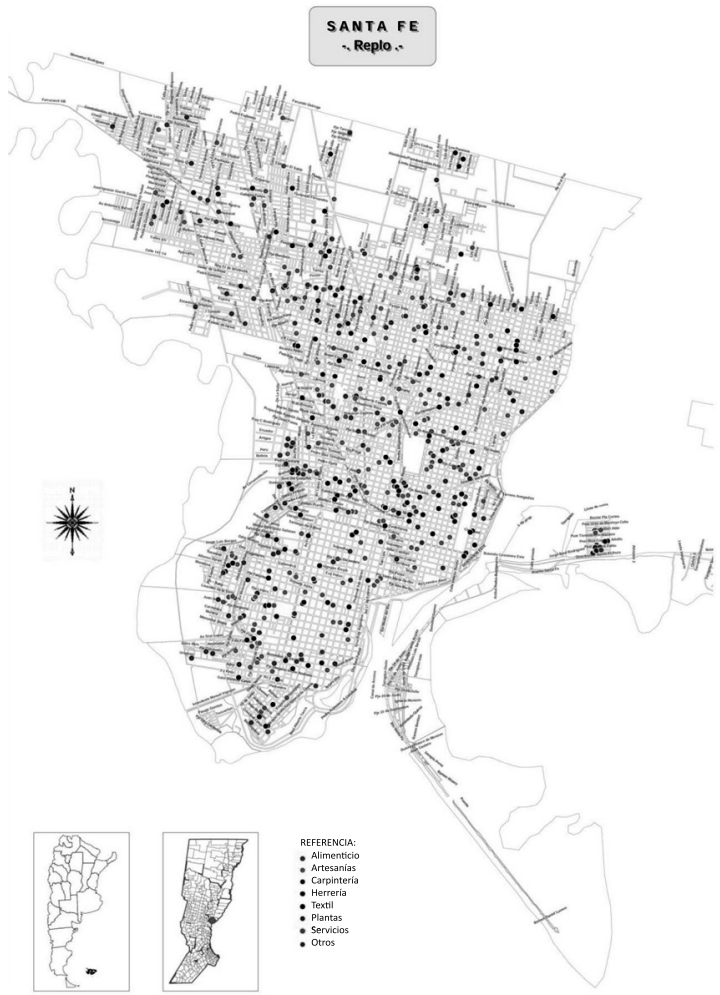

Figura 6. Distribución territorial de Emprendimientos Productivos en Santa Fe, 2014. Elaboración propia con base en datos del Registro de Emprendedores de la Municipalidad de Santa Fe. 
Luego del relevamiento efectuado y como se puede apreciar en la figura 7, podemos concluir que el núcleo de mayor importancia en cuanto a cantidad de experiencias de la Esys lo constituyen los emprendimientos productivos, el cual representa el $77 \%$ de las prácticas de esys registradas en el municipio. Consideramos que es pertinente destacar la relevancia que esta información posee para el Estado local al momento de diseñar e implementar políticas públicas para las Esys que han crecido de forma considerable en los últimos años, como lo hemos señalado en apartados interiores. Si bien se presenta como una oportunidad la articulación de políticas a través del Centro de Emprendedores de la Economía Social, el desafío es que los emprendimientos relevados puedan llegar a transformarse en emprendimientos asociativos y dejar la individualidad, condición que los hace muy vulnerables.

La Esys "tradicional” representa poco menos del $25 \%$ de las experiencias registradas en Santa Fe, lo que permite concluir que este sector necesita del apoyo del Estado en sus diferentes ámbitos (municipal, provincial y nacional), y así desarrollarse y fortalecerse como movimiento, incrementando su importancia, tanto en cantidad como calidad en su composición, apuntando a que el mismo se convierta en un pilar sumamente importante de la Esys en el municipio.

Casi la totalidad de cooperativas inscritas en Santa Fe son de trabajo. Esta representación se debe en parte a la promoción de la formalización de prácticas asociativas con preexistencia en distintos barrios de la ciudad en los últimos 10 años por parte de la $\mathrm{Mu}$ nicipalidad de Santa Fe, siendo un actor importante en la promoción de este tipo de experiencias (Tealdo, Sotto y Serafino, 2015). Entendemos que también se debe fomentar y alentar la creación de otros tipos de cooperativas (consumo, vivienda, servicios públicos, entre otros), una manera de seguir fortaleciendo y diversificando el movimiento cooperativo.

Hay que aclarar que el listado suministrado por el INAES no ofrece el rubro u objeto social de todas las mutuales, aclarándose que "en algunas entidades no figura la actividad", lo cual refleja que hay carencia de información sobre las actividades de más de la mitad de las mutuales registradas para el caso santafesino. Asimismo, las mutuales de salud y servicios sociales representan poco más del $20 \%$, lo que denota una importante presencia de este sector. Para culminar el análisis del sector, de acuerdo con los datos relevados desde 1997, no se ha conformado ninguna mutual dedicada a proveer de ayudas económicas, crédito o subsidios en el municipio.

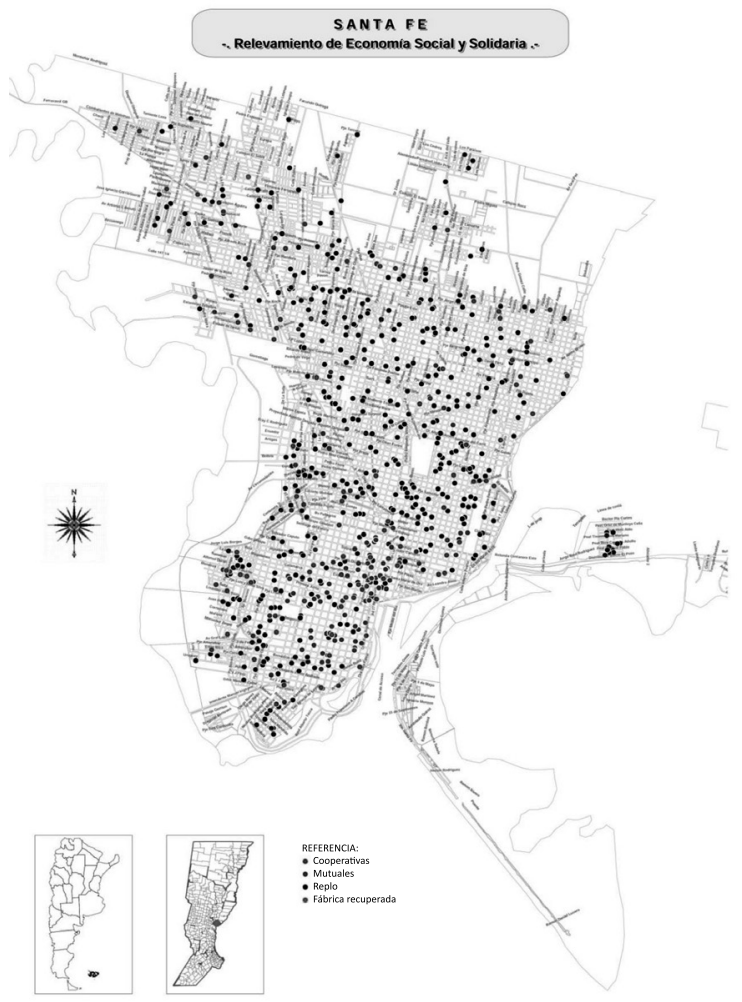

Figura 7. Distribución territorial de emprendedores productivos, cooperativas y mutuales en Santa Fe. Elaboración propia con base en datos del Registro de Emprendedores de la Municipalidad de Santa Fe y el InAEs, 2014.

\section{Consideraciones finales}

El presente trabajo es el primer relevamiento en cuanto a la centralización y sistematización de la información disponible sobre las experiencias relativas a la Esys en el municipio, debido a que no hay antecedentes de investigaciones referidas a este tipo de estudio a nivel local.

El hecho de realizar georreferenciación de las iniciativas de Esys relevadas no es solo una tarea que nos posibilita identificar el lugar donde se encuentran constituidas legalmente, sino que también es el primer paso para el diseño de una estrategia integral que nos permita elaborar una planificación para la concreción de redes solidarias con la finalidad de fortalecer un entramado con las experiencias analizadas en el presente artículo.

En Santa Fe existen diversos organismos públicos que se encuentran trabajando en este campo, entre ellos podemos mencionar, la Dirección de Economía Social y la Subsecretaría de Empleo en el ámbito municipal; la Subsecretaría de Economía Social del 
Ministerio de Desarrollo Social y la Subsecretaría de Asociativismo y Economía Social del Ministerio de la Producción, en el contexto provincial, y el Institutito Nacional de Asociativismo y Economía Social, en la instancia nacional.

Sumado a ellos, existen otras instituciones que intervienen territorialmente en la creación, fortalecimiento y promoción de prácticas de Esys. Los que han cobrado mayor visibilidad en los últimos años son las siguientes: el Foro de Economía Social y Solidaria de la Provincia Santa Fe, federaciones de cooperativas, confederaciones, institutos de promoción de la economía social, federaciones de mutuales y otros actores institucionales como la Universidad Nacional del Litoral, Universidad Tecnológica Nacional -Regional Santa Fe - y la Universidad Católica de Santa Fe.

Retomando los aportes de Euclides André Mance (2013), el mapeo institucional que acabamos de mencionar plantea un desafío en términos organizacionales e interinstitucionales para la generación y consolidación de una red de colaboración solidaria que apuntale las experiencias estudiadas para el afianzamiento de la cadena productiva de acuerdo a su objeto social y rubro. Este potencial entramado no es solo en términos productivos, sino también en los valores de la Esys que generen mayores procesos asociativos, de participación horizontal entre todos sus componentes y de compromiso por la construcción de otra economía.

Por último, consideramos que este artículo es una base importante para dilucidar la situación de las viejas y de las nuevas tradiciones de la Esys con los referentes clave de Santa Fe. Asimismo, esperamos que los resultados obtenidos se utilicen como plataforma para diseñar e implementar diversas propuestas de trabajo que permitan resolver problemáticas de las experiencias involucradas en el campo de las políticas públicas.

\section{Referencias}

Abramovich, A. y Vázquez, G. (2007). Experiencias de la Economía Social y Solidaria en la Argentina. Revista Estudios Fronterizos, 8(15), 121-145.

Bageneta, M. (2015). Del algodón a la soja. Territorio, actores y cooperativas en el Gran Chaco Argentino (1960-2010). Buenos Aires: Intercoop.

Canadian Social Economy нuв. (2009). Recuperado de http://socialeconomyhub.ca/
Cardozo, L. y Serafino, E. (2015, agosto). ¿Economía social y solidaria, estas ahí? Mapa socioterritorial basado en el Registro de Emprendedores y Productores Locales (REPlo) del Municipio de Santa Fe, Argentina. XIII Jornadas de Jóvenes Investigadores de la Asociación de Universidades del Grupo Montevideo. Universidad Nacional de la Plata, Buenos Aires.

Cardozo, L., Serafino, E., Sotto, O. y Tealdo, J. (2015). Cartografía(s) de la economía social y solidaria en el municipio de Santa Fe (Argentina). XI Jornadas de Sociología. Facultad de Ciencias Sociales, Universidad de Buenos Aires, Buenos Aires.

Colombari, B. y Molina, M. (2014). Mapeo de las experiencias de fábricas y empresas recuperadas. Revista del Observatorio Social sobre Empresas Recuperadas y Autogestionadas, 10, 60-63.

Coraggio, J. L. y Sabaté, A. (Dirs.) (2010). Emprendimientos socioeconómicos asociativos: su vulnerabilidad y sostenibilidad. Los Polvorines: Universidad Nacional de General Sarmiento.

Coraggio, J. L., Arancibia, M. y Deux, V. (2010). Guía para el mapeo y relevamiento de la economía popular solidaria en América Latina y el Caribe. Lima: GRESP-Ripess-Riless.

Gaiger, L. (2014) Conhocer globalmente: un desafío inadiável dos estudos sobre a Economía Solidária. Otra Economía, 8(14), 99-111. doi: https://doi.org/10.4013/ otra.2014.814.09

Gobierno de la Ciudad de Santa Fe. (s. f.). Economía social. Recuperado de http://www.santafeciudad.gov.ar/ciudad/economia_social.html

Hintze, S. (2007). Políticas sociales argentinas 1990-2006. En M. Vuotto (Coord.), La co-construcción de políticas públicas en el campo de la Economía Social. Buenos Aires: Prometeo Libros.

Hintze, S. (2013). Políticas públicas para otra economía. En D. Maidana y C. Valeria (Eds.), Hacia otra economía (pp. 31-39). Los Polvorines: Universidad Nacional de Sarmiento

Instituto Nacional de Estadística y Censos. República de Argentina. (Indec). (2010). Censo 2010. Recuperado de https://www.indec.gov.ar/nivel4_default.asp?id_tema_1=2\&id_tema_2=41\&id_tema_3=135

Mapeo de Economía Solidaria Uruguay. (2016). Recuperado de http://mapeoecsol.blogspot.com.ar/

Lozeco, J. (2015). La integración institucional para el desarrollo de la Economía Social y Solidaria. La expe- 
riencia del Centro de Emprendedores de la Economía Social en Santa Fe, Argentina. Revista +E, (5), 174-181

Observatorio Social sobre Empresas Recuperadas y Autogestionadas. (OSERA). (2013). Mapa de Recuperadas. Recuperado de http://empresasrecuperadas.org/mapa.php

Pastore, R. (2010). Un panorama de surgimiento de la economía social y solidaria en Argentina. Revista de Ciencias Sociales, (18), 47-74.

Projeto sies. (2014). Atlas Digital da Economia Solidária. Recuperado de http://sies.ecosol.org.br/atlas

Mance, E. (2013). Redes de colaboración solidarias. En J. L. Coraggio, J. LLaville y A Cattani (Eds.), Diccionario de la otra economía (pp. 297-303). Los Polvorines: Universidad Nacional General Sarmiento.
Ministerio de Salud y Desarrollo Social. (s. f.). Instituto Nacional de Asociativismo y Economía Social (INAES). Recuperado de http://www.inaes.gov.ar/

Vaillancourt, Y. (2010). El mapeo de la economía social y solidaria: algunos retos. Otra Economía, 7 (IV), 178-185.

Vuotto, M. (2007) El cooperativismo de trabajo y la promoción del empleo. En Vuotto, Mirta. (coord.), La coconstrucción de políticas públicas en el campo de la Economía Social (pp. 11-135). Buenos Aires: Prometeo Libros.

Tealdo, J., Sotto, O. y Serafino, E. (2015). Fortalecimiento de la (auto)gestión de cooperativas de trabajo en el marco de la economía social y solidaria en la ciudad de Santa Fe. Revista +E, (5), 188-195. 